\title{
Type I Collagen-Derived Injectable Conductive Hydrogel Scaffolds as Glucose Sensors
}

Ranjithkumar Ravichandran, J ose Gabriel Martinez Gil, Edwin J ager, J aywant Phopase and Anthony Turner

The self-archived postprint version of this journal article is available at Linköping University Institutional Repository (DiVA):

http:/ / urn.kb.se/ resolve?urn=urn:nbn:se:liu:diva- 148249

N.B.: When citing this work, cite the original publication.

Ravichandran, R., Martinez Gil, J . G., J ager, E., Phopase, J ., Turner, A., (2018), Type I CollagenDerived Injectable Conductive Hydrogel Scaffolds as Glucose Sensors, ACS Applied Materials and Interfaces, 10(19), 16244-16249. https:// doi.org/10.1021/ acsami.8b04091

Original publication available at:

https:// doi.org/ 10.1021/acsami.8b04091

Copyright: American Chemical Society

http:// pubs.acs.org/ 


\section{Type I Collagen derived injectable conductive hydrogel scaffolds as glucose sensors}

Ranjithkumar Ravichandran, ${ }^{\dagger}$ Jose G Martinez, ${ }^{\dagger}$ Edwin W H Jager, ${ }^{\dagger}$ Jaywant Phopase, ${ }^{\dagger * *}$ Anthony P F Turner ${ }^{\dagger}$

${ }^{\dagger}$ Department of Physics, Chemistry and Biology (IFM), Linköping University, SE-581 83, Linköping (Sweden)

${ }^{\ddagger}$ Department of Science and Technology, Organic Electronics, Linkoping University, Norrkoping SE-601 74, Sweden

KEYWORDS: Electroconductive hydrogel, methacrylated collagen, polypyrrole, injectable glucose sensor, diabetes.

\section{ABSTRACT:}

The advent of home blood glucose monitoring revolutionized diabetes management and the recent introduction of both wearable devices and closed loop continuous systems have impacted enormously on the lives of people with diabetes. We describe the first fully injectable soft 
electrochemical glucose sensor for in situ monitoring. Collagen, the main component of native extracellular matrix (ECM) in humans and animals, was used to fabricate an in situ gellable selfsupporting electroconductive hydrogel that can be injected onto an electrode surface or into porcine meat to detect glucose amperometrically. The study provides a proof of principle of an injectable electrochemical sensor suitable for monitoring tissue glucose levels which may, with further development, prove clinically useful in the future.

Diabetes is the fastest growing chronic disease in the World; a $56 \%$ increase in incidence is predicted by 2040, resulting in 642 million people being afflicted (International Diabetics Foundation's- IDF 2016). ${ }^{1}$ Increased blood glucose levels lead to severe complications and the disease currently afflicts around $6 \%$ of the world's population, i.e. 415 million diabetics worldwide, half of which remain undiagnosed (IDF 2016). ${ }^{1,2} 52$ million people in Europe or 8.1\% of the population have diabetes and their healthcare costs are at least double that of non-diabetics. Asia (China and India) now has the world's two largest diabetic populations (WHO 2013). ${ }^{3}$ Most importantly, there is no known reliable cure and management of the disease by monitoring and control is essential for the wellbeing of the millions of sufferers.

Biosensors, as classically defined, incorporate biological or biologically derived sensing elements that harness the exquisite specificity and sensitivity of living systems in conjunction with electronic transducers and processors, to either provide data or to directly actuate an appropriate response. The most powerful example to date, has been the evolution of glucose biosensors used in conjunction with the artificial or "bionic" pancreas. ${ }^{4}$ Enzyme electrodes for glucose detection were first successfully launched commercially in decentralized analyzers, in 1975, by the Yellow Spring Instrument Company (Ohio, USA), and then in a printed format for use by people with 
diabetes in their home, in 1987, by MediSense ${ }^{\mathrm{TM}}$ (latterly acquired by Abbott, USA). The first wearable continuous glucose monitors appeared in 2005 (Medtronic, USA). In 2012, some degree of automation for these devices was approved, first in Europe and then USA, allowing the biosensors to be used to shut off insulin delivery from an insulin pump to reduce the risk of hypoglycaemia. ${ }^{5}$ In 2017, the first closed loop insulin delivery system, (MiniMed 670G ${ }^{\mathrm{TM}}$ ) became available, which automatically adjusts the delivery of basal insulin in response to a subcutaneous glucose sensor. Another important practical innovation was the introduction of Abbott FreeStyle ${ }^{\circledR}$ Libre in 2014. This adaptation of a continuous glucose sensing needle has proved extremely popular with patients and measures glucose every minute in interstitial fluid and is scanned with a hand-held instrument. ${ }^{6}$ A needle (5mm long, $0.4 \mathrm{~mm}$ wide) is inserted under the skin using an applicator and held with adhesive pad. The device is worn on the back of the upper arm for up to 14 days. All these formats involve solid, fixed architectures and new possibilities for monitoring and tissue engineering could be facilitated by a more biocompatible, soft sensor that can be easily introduced into the body and conform more closely to tissue under investigation.

Hydrogels, 3D networks of natural polymers (collagen, gelatin, chitosan, dextran) and synthetic polymers have been used in wide range of applications such as tissue engineering, drug delivery and as biosensors. ${ }^{7,8}$ Collagen, the major component of native ECM in humans and animals, offers potential advantages such as mechanical strength and biocompatibility when fabricated in the form of hydrogels, fibers or sponges. ${ }^{7,8}$ In our previous work, we overcame the potential limitations of collagen rendering it useful as a major ECM mimetic polymer for tissue engineering and biomedical applications. ${ }^{7,}{ }^{8}$ Hydrogel-based optical and visco-elastic glucose sensors have previously been reported for use in vitro and in vivo applications. ${ }^{2}$ Injectable hydrogels as a 3D interface material for biosensing, offers potential advantages in that it can be delivered to the target 
site in a minimally invasive manner to monitor glucose levels. ${ }^{9}$ For example, fluorescently labelled injectable microbeads and hydrogel fibers for continuous glucose monitoring in vivo have been described. $^{9,} 10$ Intrinsically conducting polymers $(\mathrm{CP})^{11}$ have been extensively used in sensors, either in biosensors ${ }^{12,13}$ or as reactive sensors of any variable affecting their oxidation/reduction rate. $^{14}$ The first case includes the incorporation of enzymes such as glucose oxidase, which catalyzes the oxidation of glucose to produce an amperometric response. ${ }^{15}$ Thus, combining the properties of $\mathrm{CP}$ with an ECM mimetic polymer offers a potentially attractive approach to fabricate materials that mimic the architecture of the native ECM, while the CP provides the electronic functionality needed for an electrochemical sensor to detect bioactive molecules.

Here, we demonstrate a proof of concept for continuous glucose monitoring in vitro and in tissue (porcine meat) using collagen-based electroconductive hydrogel (ECH) as a soft sensor. By combining the advantages of electroconductive polypyrrole (PPy) and ECM mimetic collagen, an injectable double network electroconductive hydrogel, using methacrylated collagen (MAC) as a building block, has been fabricated. Combinatorial approaches have aided the development of multi-functional hydrogel matrices by linking respective reactive monomers either physically and/or chemically. ${ }^{16}$ In general, the scaffold's mechanical properties can be tuned depending on the monomer concentrations, such that the user can choose a formulation with appropriate flow properties that can be easily injected. Also, the diffusion of bioactive molecules and analytes can be controlled by the cross-linking density as a function of monomer concentration such that the hydrogel properties can be readily modulated. ${ }^{2}$ Material characterization was carried out using FTIR and oscillatory rheology to assess the structural and mechanical properties, respectively. Chronoamperometric measurements of glucose using the ECH were achieved by encapsulating glucose oxidase (GOx) inside the hydrogel matrix serving to produce a soft sensor that conforms 
to the tissue matrix. Amperometric detection at the PPy generated sufficient signal to detect relevant levels of glucose over the range 0 to $10 \mathrm{mM}$. To simulate the utility of these biocompatible ECM mimetic hydrogel sensors in practical use, the injectable ECH was administered into porcine meat for continuous measurement of glucose over 5 days using a co-axial electrode design. Our novel work can be adapted for a very wide range of analytes and opens new possibilities for both research and clinical application of in vivo biosensors.

We have previously identified some of the criteria necessary to design an injectable hydrogel. ${ }^{7}$ ${ }^{8}$ Chemical crosslinking provides unique control over manipulation of material properties such that the mechanical and structural properties can be tuned by varying the concentrations of precursor monomers. ${ }^{7}{ }^{8}$ In this paper, we have used MAC as a ECM mimetic building block and pyrrole as a monomer to form the CP. They were co-polymerized under physiological conditions using redox initiators/oxidants ammonium per sulphate (APS), iron (III) tosylate and N, N, N, Ntetramethylethylenediamine (TEMED), where methylenebis(acrylamide) (MBAA) served as crosslinking agent between methacrylate groups in MAC. Figure 1A, illustrates the fabrication route of the ECH. The ECH was spontaneously formed as a self-supporting material, after the injection, that could be molded into any desired shape and size.

The presence of PPy and collagen in the ECH hydrogel was characterized using FT-IR (Figure 1B). The peaks at $1650 \mathrm{~cm}^{-1}$ and $1560 \mathrm{~cm}^{-1}$ represent the characteristic amide I ( $\mathrm{C}=\mathrm{O}$ stretching) and amide II (N-H bending) in collagen. ${ }^{17}$ The peaks at $811 \mathrm{~cm}^{-1}$ and $1036 \mathrm{~cm}^{-1}$ are attributed to $\mathrm{C}-\mathrm{H}$ wagging and $\mathrm{C}-\mathrm{H}$ in plane stretching respectively, whereas the peak at $1685 \mathrm{~cm}^{-1}$ represents $\mathrm{C}=\mathrm{N}$ stretching in PPy. ${ }^{18}$ The presence of characteristic collagen peaks and PPy peaks in the ECH20 spectrum illustrates the formation of interpenetrating networks of MAC and PPy in our final hydrogel. The incorporation of PPy resulted in an electroactive hydrogel (Figure S1 - 
supporting information) with an electronic conductivity of $3.4 \times 10^{-3} \pm 1 \times 10^{-4} \mathrm{~S} \mathrm{~cm}^{-1}$. Furthermore, we have evaluated the rheological properties of ECH. In our earlier reports, we have shown that the introduction of methacrylate functional groups in the collagen side chains allows the user to tune the mechanical properties depending on the concentrations of MAC and cross linker. ${ }^{8}$ Figure 1C, shows the storage modulus of ECH, that can be tuned as a function of MAC content (wt\%) from $1 \mathrm{kPa}(20)$ to $3 \mathrm{kPa}(50)$. In order to use a formulation that can be easily administered in a minimally invasive manner, we chose ECH20 for our further studies. Glucose oxidase (GOx) was added into the ECH20 formulation in a syringe mixer before polymerization and the sensing properties of the resultant hydrogel was further investigated.

In order to check the sensing characteristics of the injectable glucose sensor based on ECH20, in vitro characterization in PBS buffer solution $(\mathrm{pH}=7.4)$ was performed. ECH20 containing glucose oxidase was cast on an adhesive copper tape having an area of $1 \mathrm{~cm}^{2}$ with a very narrow strip in the upper part to allow electrical connection. Figure 2A shows the chronoamperometric responses from the injectable sensor with an applied voltage of $0.6 \mathrm{~V}$ (vs. $\mathrm{Ag} / \mathrm{AgCl}$ ) when it was immersed in PBS buffer solution with different glucose concentrations. As can be seen, the current increased with increasing glucose concentration, as expected, until the sensor reached saturation. ${ }^{19}$ The current obtained during chronoamperometry in different glucose concentrations after $100 \mathrm{~s}$, produced a linear calibration curve (Figure 2B) up to a concentration of about $4 \mathrm{mM}$ glucose. The repeatability of sensor (Figure 2C) was evaluated by repeating chronoamperometric measurements from Figure 2A in 5mM glucose $\mathrm{PBS}$ buffer solution. Before each measurement, a chronoamperogram was obtained in PBS buffer solution without glucose, under the same conditions. After 10 measurements, constant performance of the sensor could be observed, with precision similar to the standard deviation of the data presented in Figure 2B under the same 
conditions. The calibration curves were obtained on different days after the generation of the ECH20 to check its stability. Figure 2D shows the calibration curves obtained after 27 days. We observed no sensing response of ECH20 during the first hour after fabrication of ECH20 (day 1), resulting in a chaotic calibration curve. This could be attributed to the slow polymerization of pyrrole which may not be complete at that time. After 24 hours post fabrication, the sensing activity of ECH20 behaved as in the chronoamperometric graph showed in Figure 2A. Figure 2D also shows that the glucose signal decreased on average with time, which could be due to the loss of material attached to the copper tape during storage. In order to select the best time for the experiment the coefficient of determination $\mathrm{R}^{2}$ (Figure 2E), sensitivity (Figure 2F) and Limit of Detection (LOD) (Figure 2G) were examined. They were obtained after linear fitting of the calibration curves using different experimental times (0.2, 20, 40, 60, 80 and $100 \mathrm{~s})$ in different glucose concentrations $(0,1,2,3,4 \mathrm{mM})$. It can be seen that both $\mathrm{R}^{2}$ and LOD improved after longer times. After only short times of potential application ( $0.2 \mathrm{~s})$, the LOD of more than $2 \mathrm{mM}$ indicated that the sensor was not useful for practical application. After 60s, the three parameters studied remained constant, with no advantage accruing for longer times. Thus, 60s was the time selected for the measurements. From our results, we found that ECH20 present higher sensitivity and similar limit of detection compared to other hydrogel-based glucose sensor. ${ }^{20}$ There is some controversy in the literature regarding hydrogen peroxide electrochemistry at PPy. Despite numerous reports about glucose sensors based on GOx immobilised in PPy, there are other reports suggesting that hydrogen peroxide electrochemistry occurs on the supporting metal or at incorporated metal nanoparticles. ${ }^{21,22,23}$ In order to elucidate the mechanism of our sensor, we followed the electrochemistry of increasing concentrations of hydrogen peroxide solution at our chemically polymerized PPy on paper in the complete absence of metal i.e. the electroactivity of 
metal did not interfere with the detection of glucose (Figure S2 - supporting information). Thus, confirmed that our system is an injectable glucose sensor with all the electrochemical sensing reactions taking place inside the gel.

ECH20 encapsulated with GOx was then injected into porcine meat. A home-made co-axial needle was used to ensure proper electrical contacts to detect glucose by chronoamperometry. The injected ECH was used to measure glucose from day 1 to day 5 in porcine meat. At day 1, the sensor response was insufficient to detect glucose, and this can be attributed to incomplete polymerization of pyrrole as already illustrated in Figure 2D. On day 2, a reasonably reliable glucose measurement could be taken. After the second day, the signal decreased (Figure 3A). It is known that the amount of glucose decreases over time in stored meat due to aerobic metabolism by microorganisms present in the meat. ${ }^{24,25}$ In order to correlate the amount of glucose present in the porcine meat with that measured by the injected $\mathrm{ECH}$, the meat samples were separately assayed using a glucose-hexokinase assay. The sensing responses from the injected ECH20 correlated with the amounts of glucose estimated from glucose-hexokinase activity 24 hours after gel injection (Figure 3A). To estimate whether the ECH20 soft sensor was still active after being injected into meat, the injected ECH20 was removed from porcine meat after 5 days and attached to a new copper tape electrode. Figure 3B shows the calibration curve of the glucose sensor in vitro in different concentrations of glucose in PBS buffer solution, post extraction of the injected ECH from meat.

In summary, predictable manipulation and fabrication of electrically responsive hydrogel scaffolds was achieved by using two different (natural/insulating and conducting) functional components. The insulating polymer provided a biocompatible 3D scaffold matrix and the CP imparted electrical conductivity to the scaffold so that it could be used as a functional material for 
biosensor applications. Utilizing the advantages of MAC (a natural polymer), PPy (conducting polymer) and GOx (glucose responsive element), an injectable ECH was fabricated using freeradical co-polymerization. The aqueous solubility of pyrrole facilitated the fabrication of a homogenous self-supporting ECH consisting of a MAC-PPy double network. The properties of the ECH were readily modulated as a function of MAC concentration such that it could not only be administered in a minimally invasive way, but also allowed sufficient glucose to diffuse inside the matrix to enable electrochemical detection via catalytic oxidation by GOx. The activity of the injectable sensor depended on electrochemical reactions occurring at PPy, and not at the electrical contacts. ECH 20, In summary, we demonstrated the first proof of concept of a collagen-derived, injectable, soft electrochemical sensor that can potentially measure analytes such as glucose in vivo. The soft electrode can conform to any tissue and provides an interface to detect redox species either directly or via enzymatic catalysis. The degradability of the injectable sensor and how it affects the sensor's function and properties will be addressed in future work. Invasiveness is reduced, since the size of the electrode is independent of the size of the puncture wound necessary to introduce the electrode in vivo. Further work on using this design for a range of clinical and wearable electronic devices and cell-based sensing applications will be explored and addressed in our future research. 


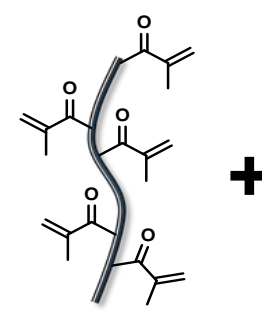

MA Collagen

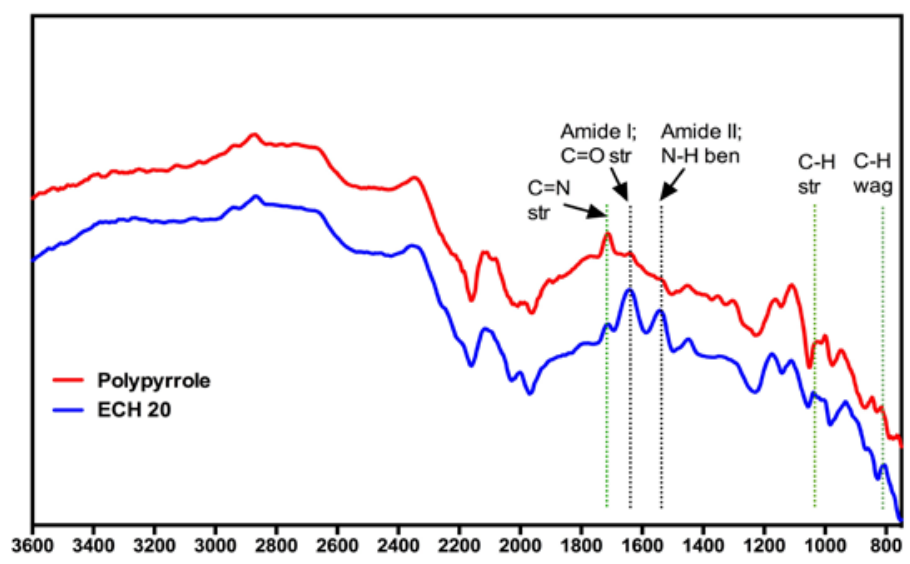

wavenumber $\left(\mathrm{cm}^{-1}\right)$

$\frac{\text { MBAA, APS / TEMED }}{\text { PBS (pH 7.4) }}$

Pyrrole
PN network of collagen and polypyrrole

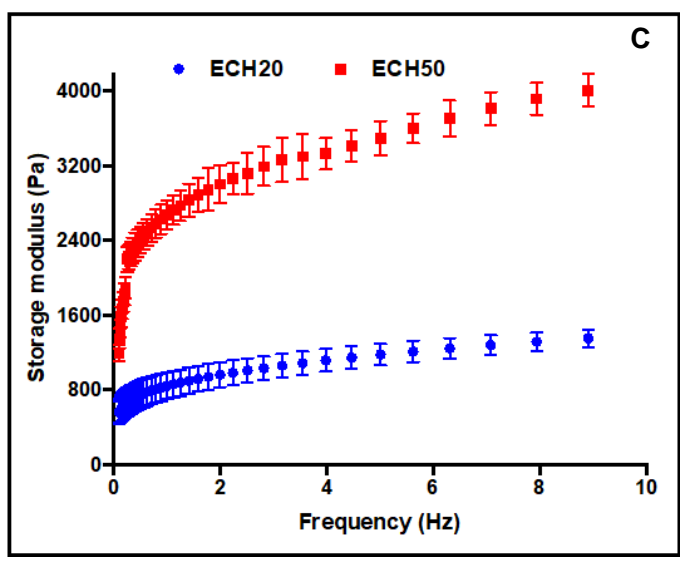

Figure 1. (A) Design and fabrication of an electroconductive hydrogel (ECH) from MAC and pyrrole as pre-cursors. (B) FT-IR spectra of PPy film and ECH, (C) Mechanical properties of ECH characterized using rheology. 

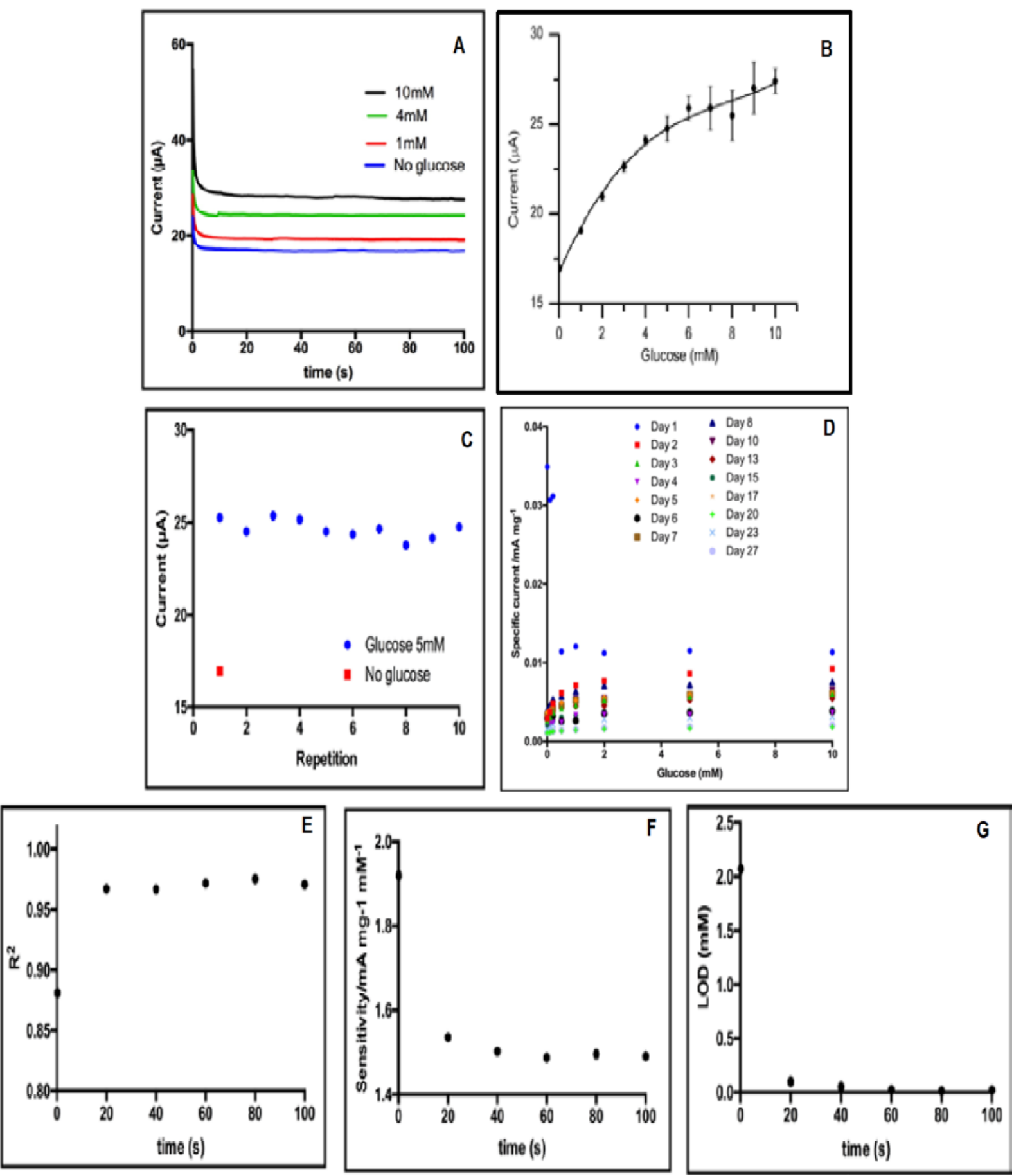

Figure 2: ECH20 containing glucose oxidase was cast on an adhesive copper tape having an area of $1 \mathrm{~cm}^{2}$ with a very narrow strip in the upper part to allow electrical connection. (A) Chronoamperometric responses obtained from such ECH20 injectable glucose sensor with an applied voltage of $0.6 \mathrm{~V}$ (vs Ag/AgCl) immersed in PBS buffer solution (pH=7.4) with different 
glucose concentrations (0, 1, $410 \mathrm{mM})$. (B) Calibration curve of the ECH20 injectable glucose sensor obtained from the current after $100 \mathrm{~s}$ (experimental conditions as in A). (C) Repeatability study: $0.6 \mathrm{~V}$ vs $\mathrm{Ag} / \mathrm{AgCl}$ was applied for $200 \mathrm{~s}$ to the ECH20 injectable glucose sensor, in PBS buffer containing $5 \mathrm{mM}$ glucose; between experiments, the same potential $(0.6 \mathrm{~V})$ was applied for the same time (200 s) in PBS solution without glucose. (D) Calibration curves obtained on different days starting just after polymerization (day 1 ). (E) The coefficient of determination $\mathrm{R}^{2}$ was obtained after linear fitting (using data $0 \mathrm{mM}$ glucose to $4 \mathrm{mM}$ glucose) of the calibration curves when the potential of $0.6 \mathrm{~V}$ (vs $\mathrm{Ag} / \mathrm{AgCl}$ ) was held for different experimental times (0.2, 20, 40, 60, $80100 \mathrm{~s}$ ). (F) Sensitivity obtained after linear fitting (using data $0 \mathrm{mM}$ glucose to $4 \mathrm{mM}$ glucose) from the calibration curves when the potential of $0.6 \mathrm{~V}$ (vs $\mathrm{Ag} / \mathrm{AgCl}$ ) was held for different experimental times. $(\mathrm{G}) \mathrm{LOD}=(\mathrm{S} / \mathrm{N}=3)$ obtained after linear fitting (using data $0 \mathrm{mM}$ glucose to $4 \mathrm{mM}$ glucose) from the calibration curves when the potential of $0.6 \mathrm{~V}$ (vs $\mathrm{Ag} / \mathrm{AgCl}$ ) was kept for different experimental times.
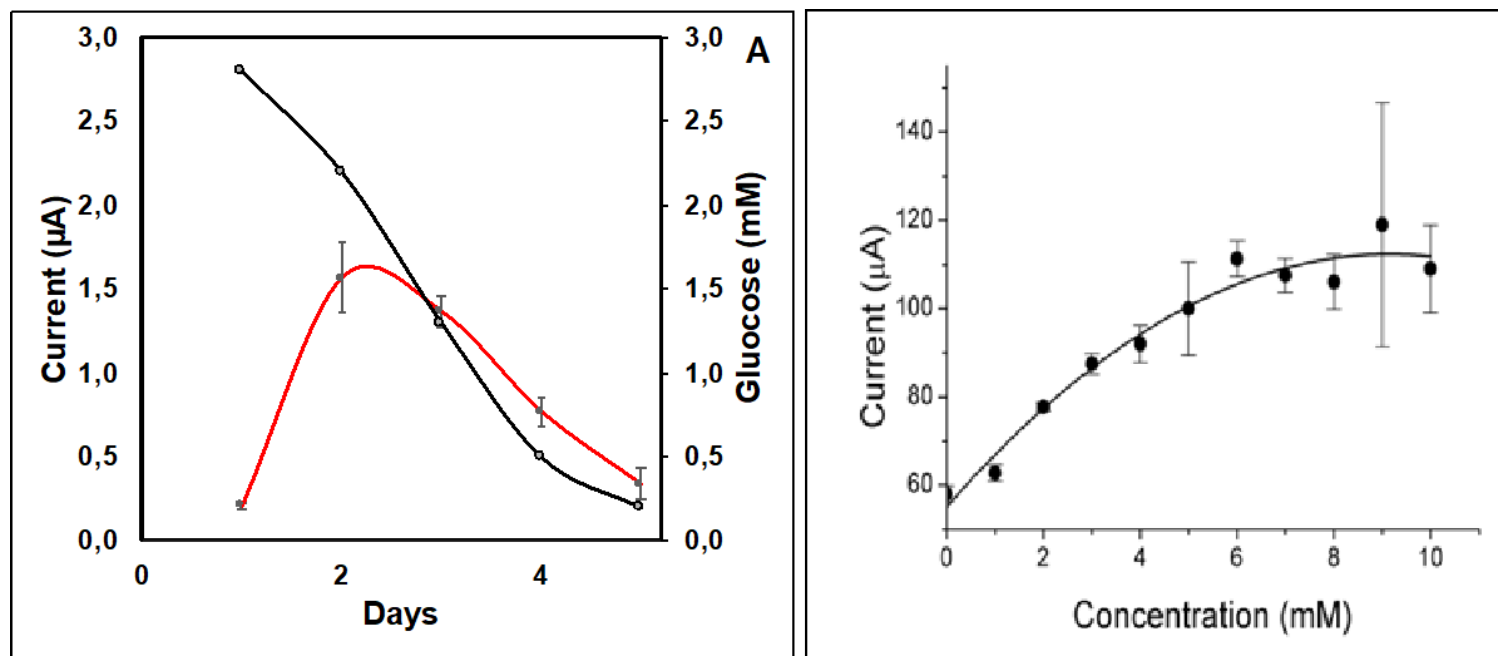

Figure 3: (A) Measured current from the chronoamperometric responses obtained from ECH20 injected into a porcine meat piece with an applied voltage of $0.6 \mathrm{~V}$ (vs $\mathrm{Ag} / \mathrm{AgCl})$, after $100 \mathrm{~s}$ on 
different days after injection; day 1 (red line) and gradual decrease of glucose concentration estimated from glucose-hexokinase activity at $4^{\circ} \mathrm{C}$ (black line). (B) Calibration curve obtained from an ECH20 sensor after extraction from a porcine meat piece obtained by applying a constant potential of $600 \mathrm{mV}$ vs $\mathrm{Ag} / \mathrm{AgCl}$ for $100 \mathrm{~s}$ in different $\mathrm{PBS}$ solutions with different glucose concentrations.

Notes

The authors disclose no financial competing interests.

\section{AUTHOR INFORMATION}

\section{Corresponding Author}

jaywant.phopase@liu.se

\section{Author Contributions}

The manuscript was written through contributions of all authors. All authors have given approval to the final version of the manuscript. JP and APFT designed and planned the research.

\section{ACKNOWLEDGMENT}

This work was supported by the Swedish Research Council grant (dnr 621-2012-4286) to JP, CeNano PhD student salary grant to JP. Carl Tryggers Stifelsen grant nr. CTS16:207 to JGM and EWHJ, Swedish Research Council grant nr. VR-2014-3079 to EWHJ. APFT gratefully acknowledges funding from the Swedish Research Council VR 2015-04434 DIABETSENS.

\section{ABBREVIATIONS}

Extra Cellular Matrix, ECM; CP, Conducting Polymer; ECH, Electro Conductive Hydrogel; PPy, PolyPyrrole; APS, Ammonium Per Sulfate; LOD, Limit Of Detection; PBS, Phosphate 
Buffer Saline; MAC, Methacrylated Collagen; TEMED, N, N, N, N-tetramethylethylenediamine; MBAA, N, N’-methylenebis(acrylamide)

\section{SUPPORTING INFORMATION}

All experimental details, hydrogel fabrication and characterization, Electrochemical characterization and supporting figures.

\section{REFERENCES}

(1) Browne, J. L.; Holmes-Truscott, E.; Ventura, A. D.; Hendrieckx, C.; Pouwer, F.; Speight, J. Cohort profiles of the cross-sectional and prospective participant groups in the second Diabetes MILES-Australia (MILES-2) study. BMJ Open 2017, 7, e012926.

(2) Chen, C.; Zhao, X. L.; Li, Z. H.; Zhu, Z. G.; Qian, S. H.; Flewitt, A. J. Current and Emerging Technology for Continuous Glucose Monitoring. Sensors (Basel) 2017, 17, 182.

(3) Rhee, E. J. Diabetes in Asians. Endocrinology and Metabolism (Seoul) 2015, 30, 263-269.

(4) Newman, J. D.; Turner, A. P. Home blood glucose biosensors: a commercial perspective. Biosensors \& bioelectronics 2005, 20, 2435-2453.

(5) McAdams, B. H.; Rizvi, A. A. An Overview of Insulin Pumps and Glucose Sensors for the Generalist. Journal of Clinical Medicine 2016, 5, 5.

(6) Bruen, D.; Delaney, C.; Florea, L.; Diamond, D. Glucose Sensing for Diabetes Monitoring: Recent Developments. Sensors (Basel) 2017, 17, 1866.

(7) Ravichandran, R.; Islam, M. M.; Alarcon, E. I.; Samanta, A.; Wang, S.; Lundstrom, P.; Hilborn, J.; Griffith, M.; Phopase, J. Functionalised type-I collagen as a hydrogel building block 
for bio-orthogonal tissue engineering applications. Journal of Materials Chemistry B 2016, 4, 318-326.

(8) Ravichandran, R.; Astrand, C.; Patra, H. K.; Turner, A. P. F.; Chotteau, V.; Phopase, J. Intelligent ECM mimetic injectable scaffolds based on functional collagen building blocks for tissue engineering and biomedical applications. RSC Advances 2017, 7, 21068-21078.

(9) Shibata, H.; Heo, Y. J.; Okitsu, T.; Matsunaga, Y.; Kawanishi, T.; Takeuchi, S. Injectable hydrogel microbeads for fluorescence-based in vivo continuous glucose monitoring. Proceedings of the National Academy of Sciences of the United States of America 2010, 107, 17894-17898.

(10) Heo, Y. J.; Shibata, H.; Okitsu, T.; Kawanishi, T.; Takeuchi, S. Long-term in vivo glucose monitoring using fluorescent hydrogel fibers. Proceedings of the National Academy of Sciences of the United States of America 2011, 108, 13399-13403.

(11) Otero, T. F.; Martinez, J. G. Electro-chemo-biomimetics from conducting polymers: fundamentals, materials, properties and devices. Journal of Materials Chemistry B 2016, 4, 20692085.

(12) Gerard, M.; Chaubey, A.; Malhotra, B. D. Application of conducting polymers to biosensors. Biosensors \& bioelectronics 2002, 17, 345-359.

(13) Ahuja, T.; Mir, I. A.; Kumar, D.; Rajesh.Biomolecular immobilization on conducting polymers for biosensing applications. Biomaterials 2007, 28, 791-805.

(14) Otero, T. F.; Martinez, J. G. Physical and chemical awareness from sensing polymeric artificial muscles. Experiments and modeling. Progress in Polymer Science 2015, 44, 62-78. 
(15) Simionescu, C. I.; Grovu-Ivanoiu, M.; Cianga, I.; Grigoras, M.; Duca, A.; Cocârla, I. Electrochemical polymerization of some monomers with schiff's base structure. A voltammetric study. Macromolecular Materials and Engineering 1996, 239, 1-12.

(16) Buwalda, S. J.; Vermonden, T.; Hennink, W. E. Hydrogels for Therapeutic Delivery: Current Developments and Future Directions. Biomacromolecules 2017, 18, 316-330.

(17) Vidal, B.C.; Mello, M. L. S. Collagen type I amide I band infrared spectroscopy. Micron 2011, 42, 283-289.

(18) Nicho, M. E.; Hu, H. Fourier transform infrared spectroscopy of polypyrrole composite coatings. Solar Energy Materials and Solar Cells 2000, 63, 423-435.

(19) Jia, W. Z.; Wang, K.; Xia, X.H. Elimination of electrochemical interferences in glucose biosensors. Trends in Analytical Chemistry:TRAC 2010, 29, 306-318.

(20) Park, H.; Lee, J.; Kim, D.; Koh, Y.; Cha, J. Hydrogel-based electrochemical sensor for noninvasive and continuous glucose monitoring. Proceedings of SPIE 2017, 10324, 1032405(1-9).

(21) Bélanger, D.; Nadreau, J.; Fortier, G. Electrochemistry of the polypyrrole glucose oxidase electrode. Journal of Electroanalytical Chemistry and Interfacial Electrochemistry 1989, 274, 143-155.

(22)Arslan, F.; Ustabas, S.; Arslan, H. An Amperometric Biosensor for Glucose Determination Prepared from Glucose Oxidase Immobilized in Polyaniline-Polyvinylsulfonate Film. Sensors (Basel) 2011, 11, 8152-8163. 
(23) Selvaraju, T.; Ramaraj, R. Electrocatalytic reduction of hydrogen peroxide at nanostructured copper modified electrode. Journal of Applied Electrochemistry 2009, 39, 321327.

(24) Favre, E.; Pugeaud, P.; Péringer, P. Automated HPLC monitoring of glucose, glutamine, lactate and alanine on suspended mammalian cell reactors. Biotechnology Techniques 1990, 4, 315-320.

(25) Umuhumuza, L. C.; Sun, X. Rapid detection of pork meat freshness by using L- cysteinemodified gold electrode. European Food Research and Technology 2011, 232, 425-431. 
Table of contents

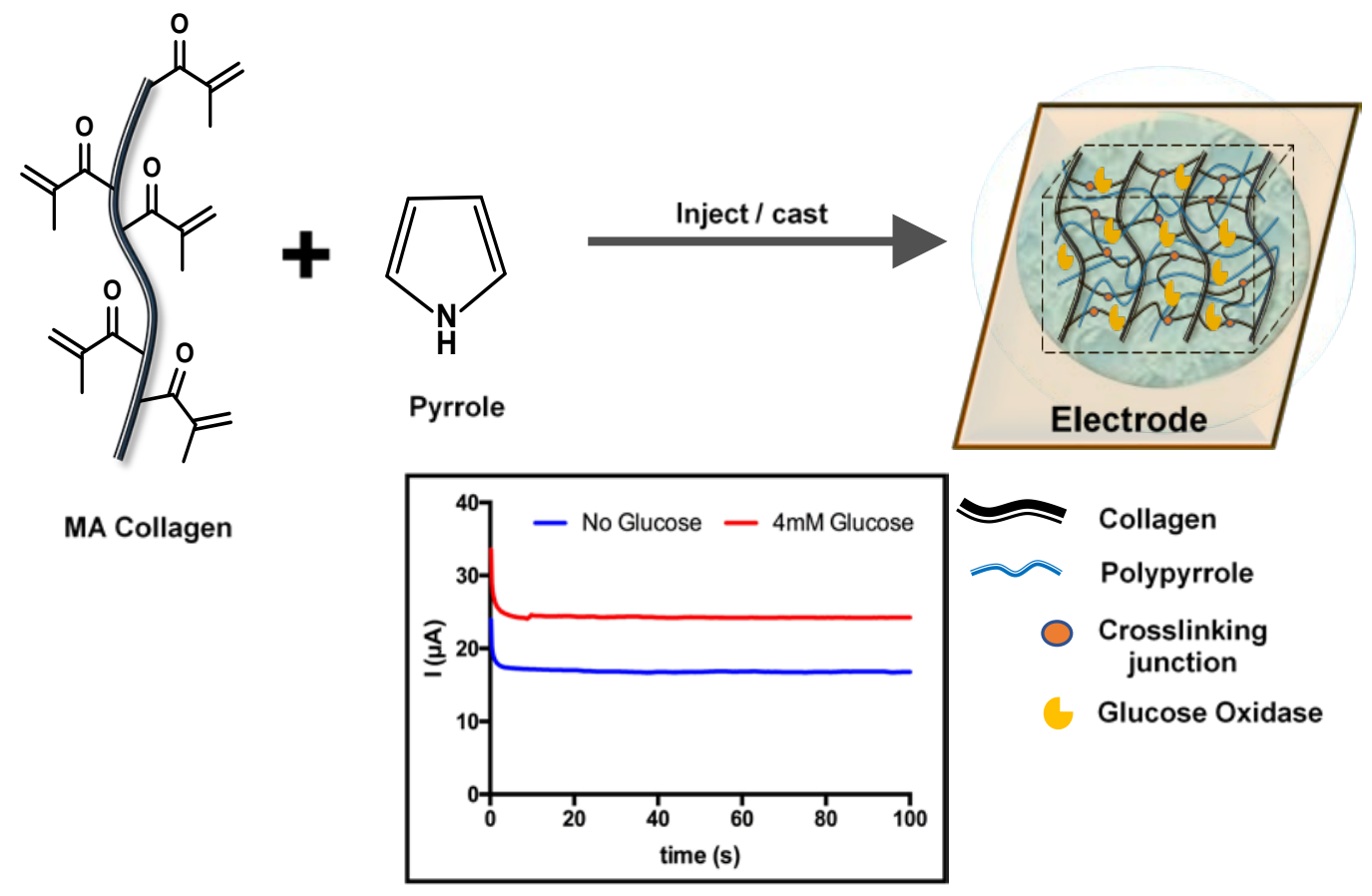

In situ gellable electro-conductive collagen hydrogel that can be injected on electrode surface or into the porcine meat to detect glucose by chronoamperometric measurements 


\section{Supporting information}

\section{Type I Collagen derived injectable conductive hydrogel scaffolds as glucose sensors}

Ranjithkumar Ravichandran, ${ }^{\dagger}$ Jose G Martinez, $^{\dagger}$ Edwin W H Jager, ${ }^{\dagger}$ Jaywant $^{\text {Phopase, }}{ }^{\dagger \neq *}$ Anthony P F Turner ${ }^{\dagger}$

${ }^{\dagger}$ Department of Physics, Chemistry and Biology (IFM), Linköping University, SE-581 83, Linköping (Sweden)

${ }^{\ddagger}$ Department of Science and Technology, Organic Electronics, Linkoping University, Norrkoping SE-601 74, Sweden

* Email: jaywant.phopase@liu.se 


\section{Experimental Section}

\section{Materials}

D (+)- Glucose anhydrous for biochemistry (from Merck), Glucose Oxidase (GOx) from Aspergillus niger, Type VII, lyophilized powder, $\geq 100,000$ units/g solid (without added oxygen, from Sigma-Aldrich) were used as received. Fe(III) Tosylate (From Heraeus Precious Metals GmbH \& Co.), poly (ethylene glycol) methyl ether methacrylate (from Sigma-Aldrich), poly (ethylene glycol) dimethacrylate (from Sigma-Aldrich) were used as received and mixed for 20 minutes under stirring with a proportion of 70/15/15 (\%w) in the respective order resulting in an oxidant mixture. Pyrrole (from SAFC®, acquired from Sigma-Aldrich) was distilled under vacuum prior to use and stored at $-20^{\circ} \mathrm{C}$. Ultrapure water was obtained from Milli-Q Plus water equipment and used for the experiments.

\section{Hydrogel fabrication and characterization}

Synthesis of MAC is reported in our previous paper. ${ }^{1}$ Stock concentration of MAC (dissolved in PBS) buffer and pyrrole were mixed in weight ratio of (1:1) with other reacting components homogenously using our syringe mixing procedure. ${ }^{1}$ Briefly, oxidant mixture $80 \mu \mathrm{L}$, MBAA (2wt $\%), 20 \mu \mathrm{L}$ APS (10wt $\%$ ) and 5 $\mathrm{LL}$ TEMED was added to MAC-pyrrole solution and mixed thoroughly before injecting or casting into any mould. The hydrogels obtained were named according to the wt\% ratio of MAC as ECH20 and ECH50. Polypyrrole films were prepared using the same method without adding MAC. Pristine polypyrrole film and ECH20 hydrogel were dried and ATR-FTIR (VERTEX FTIR spectrometer, MA, USA) spectra were recorded from 400 to $4000 \mathrm{~cm}^{-1}$ at $4 \mathrm{~cm}^{-1}$ resolution. Rheological measurements of ECH20 and ECH50 were performed using parallel plate rheometry (DHR 2 rheometer, TA instruments, Inc., UK). 
The gap was adjusted from the original sample height, compressing the sample to reach $0.03 \mathrm{~N}$; bulk modulus (G') and viscous modulus (G') were recorded $0.1-25 \mathrm{~Hz}$ at $25^{\circ} \mathrm{C}$ using $8 \mathrm{~mm}$ aluminium plate geometry $(n=3)$. Glucose Oxidase was encapsulated into the hydrogels before crosslinking using the syringe mixing procedure.

The conductivity of the ECH20 hydrogel was measured by filling a plastic tube having a length of $8.01 \pm 0.08 \mathrm{~mm}$ and an inner diameter of $3.00 \pm 0.03 \mathrm{~mm}$ with ECH 20. Electrical connections were performed using copper tape on both ends of the tube. The four points probe test was followed, using a power source Aim TTi PL303-P to apply electrical current and a digital multimeter Multimetrix ${ }^{\circledR}$ DMM220 to measure the potential.

\section{Electrochemical characterization}

All the electrochemical experiments were performed in a glass, single compartment threeelectrode electrochemical cell, connected to a Compactstat potentiostat-galvanostat from Ivium Technologies (Eindhoven, The Netherlands) controlled by a personal computer through IviumSoft 2.756 [3] software. A gold electrode having $2 \mathrm{~cm}^{2}$ of surface area was used as counter electrode. The working electrode was a copper tape covered with ECH20: 9.75-10.12 mm length and 11.90-12.15 mm width. The electrical connection was achieved through a narrow strip of the same tape to keep the submerged area of copper constant. Although the copper presented some electro activity, the current did not change with different glucose concentrations. Further experiments on the electrochemistry of hydrogen peroxide at the gel in the total absence of metal confirmed that the glucose response was due to the ECH20 sensing properties. The mass of ECH20 ranged from 69.3 to $85 \mathrm{mg}$. The reference electrode was a BASi MF-2052 Ag/AgCl (3M $\mathrm{KCl}$ ) electrode. All the experiments were performed at $22^{\circ} \mathrm{C}$ (room temperature) $(\mathrm{n}=3)$. 
In-tissue injection and glucose monitoring

In order to perform the glucose monitoring experiments in tissue, a home-made co-axial needle was prepared as follows: first, $1 \mathrm{~cm}$ diameter stainless-steel tube with external thread was inserted in the meat piece by pressing the meat (fresh pork loin, bought from local supermarket). Then, a stainless-steel needle (diameter 20G) was covered in the upper part (leaving $2 \mathrm{~cm}$ uncovered) with Parafilm ${ }^{\circledR}$ to avoid shortcuts with the outer tube and, inserted through the outer needle until the Parafilm ${ }^{\circledR}$ touched the meat. Then, using the syringe the ECH20 was injected in the meat. The electrical connections of the two separate parts (inner needle acting as working electrode and outer tube acting as reference and counter electrodes) were achieved using two crocodile clips.

Meat sample homogenization for glucose detection by glucose-hexokinase assay

The glucose content in porcine meat samples was assayed according to previously described methods. ${ }^{2,3}$ Briefly, one gram portion of porcine meat (obtained from local supermarket) was chopped up with a razor blade and homogenized in $2.5 \mathrm{~mL}$ of $6 \%(\mathrm{w} / \mathrm{v})$ perchloric acid and hydrolyzed with amyloglucosidase from Aspergillus niger (Sigma) for 2 hours followed by centrifugation for $15 \mathrm{~min}$. Clear supernatant was used for measurement of the glucose content in porcine meat using glucose-hexokinase assay kit (Sigma).

\section{Electroactivity of ECH20}

The electroactivity of ECH20 showed (Figure S1) a clear reduction peak can be observed at -0.2 $\mathrm{V}$ and a oxidation one at $0.0 \mathrm{~V}$. 


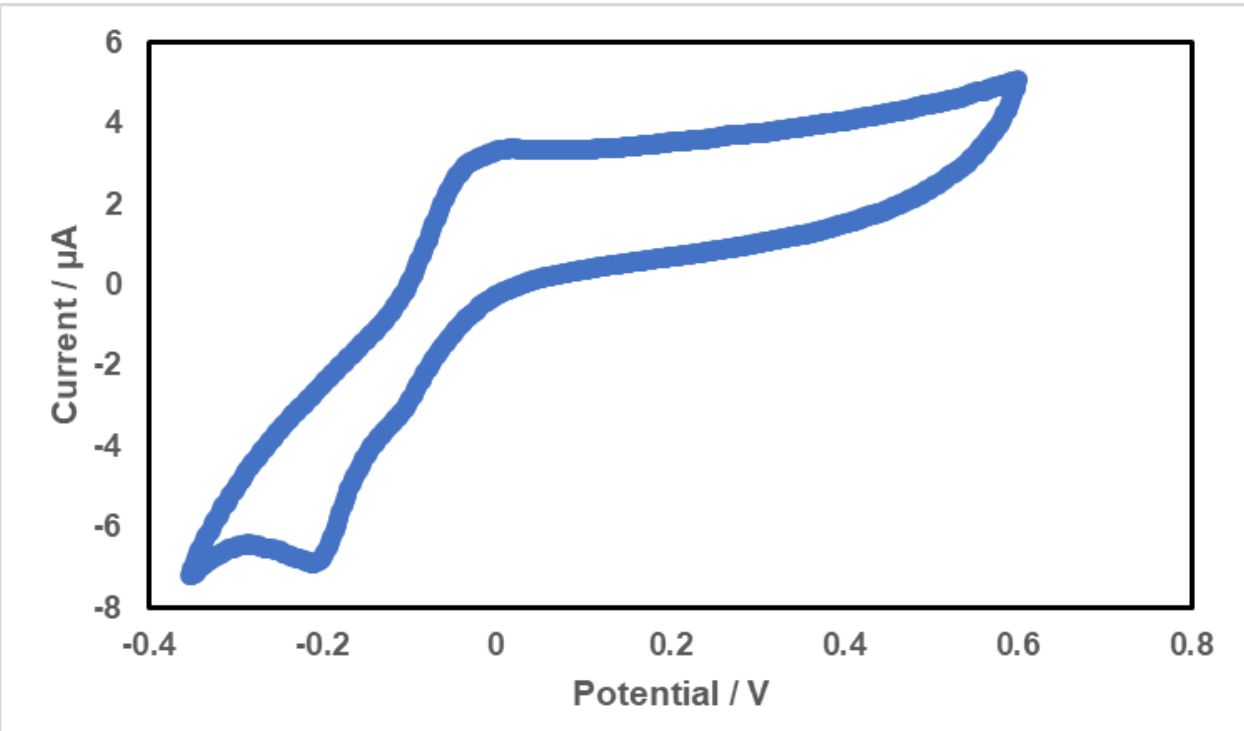

Figure S1: Cyclic voltammogram obtained from ECH hydrogel inmersed in PBS buffer solution $(\mathrm{pH}=7.4)$ at $10 \mathrm{mV} \mathrm{s}^{-1}$.

\section{Hydrogen Peroxide detection on a Polypyrrole (PPy) film}

In order to check the activity of hydrogen peroxide on PPy, we have deposited PPy into a piece of paper ( $80 \mathrm{gm}^{-2}$ ) having 48.31 x $24.38 \mathrm{~mm}$ by chemical polymerization. It was submerged into $10 \mathrm{~mL}$ of water plus $700 \mu \mathrm{L}$ of oxidant mixture, $40 \mu \mathrm{L}$ APS (10wt $\%), 10 \mu \mathrm{L}$ TEMED and $700 \mu \mathrm{L}$ of pyrrole for 22 hours at room temperature. After that, the PPy coated paper was washed thoroughly with abundant water. A smaller sample having 48.31 x $6.15 \mathrm{~mm}$ was painted with a strip of nail polish (Idum ${ }^{\circledR}$ ) approximately in the middle of its length to avoid capillarity as much as possible. Then it was clamped in the upper end with a metallic clamp and immersed in PBS solution below the paint strip. Chronoamperometric measurements were maintained in the same conditions as for sensing glucose mentioned in main text, and we have added hydrogen peroxide to the PBS solution that resulted in calibration curve as depicted in Figure S2. 


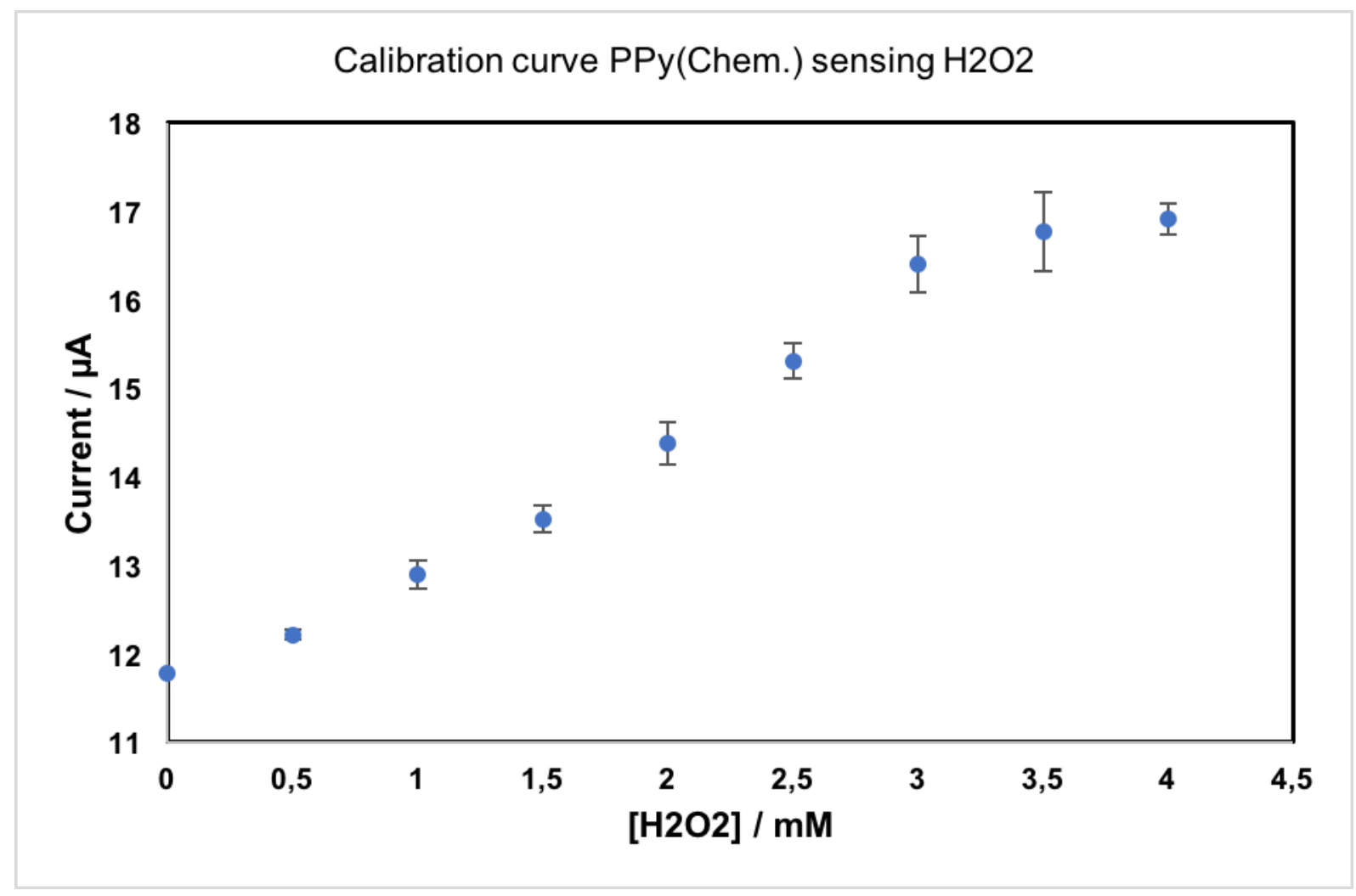

Figure S2: Electrochemical detection of hydrogen peroxide $\left(\mathrm{H}_{2} \mathrm{O}_{2}\right)$ by chemically polymerized PPy film.

\section{References}

(1) Ravichandran, R.; Astrand, C.; Patra, H. K.; Turner, A. P. F.; Chotteau, V.; Phopase, J. Intelligent ECM mimetic injectable scaffolds based on functional collagen building blocks for tissue engineering and biomedical applications. RSC Advances 2017, 7, 21068-21078.

(2) Jerez, N. C.; Calkins, C. R.; Velazco, J. Prerigor injection using glycolytic inhibitors in lowquality beef muscles. Journal of Animal Science 2003, 81, 997. 
(3) Wilborn, B. S.; Kerth, C. R.; Owsley, W. F.; Jones, W. R.; Frobish, L. T. Improving pork quality by feeding supranutritional concentrations of vitamin D3. Journal of Animal Science 2004, 82, 218. 